\title{
The impact of acute care clinical practice guidelines on length of stay: A closer look at some conflicting findings
}

\author{
Moriah Ellen $^{1,2,3}$, G. Ross Baker ${ }^{4}$, Adalsteinn Brown ${ }^{4,5}$ \\ 1. School of Management and Marketing, Jerusalem College of Technology, Israel. 2. Israeli Centre for Technology \\ Assessment in Health Care, Gertner Institute for Epidemiology and Health Policy Research, Israel. 3. Program in Policy \\ Decision Making, McMaster University, Canada. 4. Institute of Health Policy, Management and Evaluation, Faculty of \\ Medicine, University of Toronto, Canada. 5. Dalla Lana School of Public Health, University of Toronto, Canada.
}

Correspondence: Moriah Ellen. Address: Ha-Va'ad ha-Le'umi St 21, Jerusalem, 93721, Israel. E-mail:

Moriah.ellen@gmail.com

Received: November 27, 2013

Accepted: January 1, 2014

Online Published: February 13, 2014

DOI : $10.5430 /$ jha.v3n4p25

URL: http://dx.doi.org/10.5430/jha.v3n4p25

\section{Abstract}

Systematic reviews have found that clinical practice guidelines (CPGs) are associated with lower lengths of stay (LOS), but a secondary analysis of Ontario acute care hospitals found few significant relationships between CPGs and LOS. This research explored possible reasons for these findings and what other factors may impact the CPG-LOS relationship. Semi-structured interviews were conducted with staff from nine hospitals whose jobs dealt with developing, implementing, monitoring, updating, or evaluating CPGs. Interviews were analyzed utilizing methods outlined by Aurebach. A variety of leaders and hospital types were represented. Five main factors influencing relationships between CPGs and LOS were identified: 1) the purpose of implementation, 2) evidence base for CPG content and selection, 3) health care professionals' response to change and compliance, 4) dissemination strategies, and 5) organizational support and resources. The interviews suggested possible reasons why CPGs are not realizing their full potential impact on LOS in Ontario hospitals, ranging from poor compliance to resistance from health care providers. CPGs themselves are not perceived to be the reason for ineffectiveness; rather, organizational- and individual-level barriers seem to be the causes.

\section{Key words}

Clinical practice guidelines, Hospital efficiency, Length of stay, Canada

\section{I ntroduction}

Hospital administrators remain under relentless pressures to contain costs while expanding services and improving outcomes. Reducing length of stay (LOS) is a common goal, and there are a number of different ways to reduce LOS: through reduced capacity, increased integration, incentives, the utilization of clinical practice guidelines (CPGs), or a combination of these methods ${ }^{[1-3]}$. CPGs remain a common method in reducing hospital LOS ${ }^{[4]}$. CPGs are "systematically developed statements to assist practitioner and patient decisions about appropriate health care for specific clinical circumstances" ${ }^{[5]}$. CPGS are meant to streamline care, to reduce duplication and in-hospital complications, and to standardize clinicians approaches to delivering care ${ }^{[6,7]}$. In theory, CPGs should help move patients more quickly and 
safely through the hospital experience. But does the theory translate to practice? Systematic reviews have found that CPGs can reduce $\operatorname{LOS}^{[4,8]}$. A recent Cochrane review that included 27 studies involving 11,398 participants examined the effect of clinical pathways on $\operatorname{LOS}^{[4]}$. Of the 27 studies that were included, thirteen of the studies were conducted in the United States, four in Australia, three in Japan, two in the United Kingdom, two in Canada, and one each in Norway, Taiwan and Thailand (for more details on study characteristics, please see Rotter et al., 2010). The review found that in most studies significant reductions in length of stay were reported (variation in design and settings prevented the statistical pooling of results) ${ }^{[4]}$. However, a secondary analysis using Ontario acute care hospital data found few significant relationships between CPGS and LOS ${ }^{[9]}$. Given these conflicting findings, this research was conducted to explore the relationship between CPGs and LOS in the acute care hospital sector in Ontario, Canada using qualitative methods.

There are a number of reasons why the use of CPGs may be associated with different efficiency outcomes. These reasons can be clustered into two main categories, the first being those associated with the CPG itself. Qualities of the guideline itself can facilitate or hinder its effectiveness, and studies have shown that the quality of guidelines is often moderate ${ }^{[10,11]}$. "The potential benefits of practice guidelines are only as good as the quality of the practice guidelines themselves" ${ }^{[12]}$. Many of these aspects have been identified by international experts and incorporated into the AGREE tool (i.e., credibility, quality, and recommendations that are practical, feasible and evidence based) ${ }^{[13-17]}$. The AGREE tool, which now has a second version in place, is meant to evaluate the quality of CPGs, address potential biases of guideline development and ensure that the guideline recommendations are feasible, and internally and externally valid ${ }^{[12,18]}$.

The second group of factors influencing the effectiveness of a CPG are external issues to the CPG itself such as organizational level characteristics or implementation strategies. Barriers to effective implementation of CPGs can occur at a number of levels: the patient, the health care professional, the unit, the organization, or the environment ${ }^{[19]}$. In extrapolating from previous reviews, Grol and Grimshaw identify three clusters of barriers to implementing change ${ }^{[20]}$.

- The organizational context or practice environment: this includes organizational constraints such as lack of human resources and time; patients' expectations (e.g., patients want certain forms of treatment not in compliance with the CPG); a lack of funding or reimbursement; and a liability risk - there may be increased complaints.

- The social context including current standards of practice, professionals' training, the opinions of leaders, and the advocacy of various groups.

- The professional context or the knowledge, attitudes, and beliefs of the professionals: this includes the large amount and rate of information they are exposed to, the feeling that they need to do something to solve the problem, their self-confidence, and the clinical uncertainty in certain situations.

Numerous systematic reviews have examined different development and implementation strategies to ensure successful CPG implementation yet we are still unclear as to which strategies work best in different circumstances ${ }^{\text {[21-25] }}$. The difficulty in determining which implementation strategies work and creating a link between CPG usage and decreased LOS may be because CPGs "are either not well constructed, not properly implemented, or both" ${ }^{[26]}$. The purpose of this study is to find out what factors positively or negatively affect the CPG-LOS relationship in Ontario hospitals.

\section{Methods}

In-depth, semi-structured telephone interviews were conducted in hospitals in one Canadian province (Ontario). Semi-structured interviews were the chosen method, in this case because of the exploratory nature of the study. Semistructured interviews allow participants to respond freely, to focus on the area that they perceive as most influential and applicable to their organization, and permit the interviewer to explore issues that may be of interest but that are not specifically mentioned in the interview guide ${ }^{[27,28]}$. 


\subsection{Recruitment}

A purposive sample ${ }^{[29]}$ was selected of individuals who were actively engaged in CPG development and implementation. We were interested in conducting this study in acute care hospitals since the data from one of the systematic reviews, and from the secondary data analysis, were from acute care hospitals and therefore we wanted to continue to explore the relationship in a similar setting. Furthermore, we wanted to learn from organizations that had already implemented numerous CPGs and therefore had more experience to draw upon during the interview process. Finally, we wanted to speak to the individuals responsible for CPGs since their insight into the process and outcomes could assist others in the CPG implementation process. A member of the Executive Group of the Utilization Managers' Network of Ontario (UMNO), an allied group of the Ontario Hospital Association, provided contacts within the Ontario acute care hospital sector who have had experience with CPGs. The individuals interviewed were from Ontario hospitals and dealt with one or more of the following responsibilities: developing, implementing, monitoring, updating, or evaluating CPGs.

The participants were recruited by e-mail, mail, and phone. Once initial contact was established, a cover letter and consent form were sent out. When consent had been obtained from all interviewees, an interview time and date was established that was convenient for each individual interviewee. Permission was asked at the start to tape-record the interview, and all participants agreed. We ensured confidentiality of all records and interviewee identities were protected.

\subsection{Interview and analysis}

The interview schedule included basic demographics (i.e., age and education). Questions were developed based on the AGREE tool, pre-existing literature reviews, and a discussion with an expert in the field. It was made clear to the interviewees that when the term "CPG" was used, it could also refer to related terms such as best practice guidelines or pathways. If their organization utilized a different terminology, it was first clarified with the researcher to ensure the interview was applicable. Each interview took about one hour. A total of nine interviews in nine different hospitals were conducted; the subjects included eight registered nurse leaders and one non-health-care professional.

The interviews were tape-recorded and transcribed. Interviews were conducted until thematic saturation was reached. There are no specific guidelines for selecting sample sizes for studies based on purposive sampling, and the sample size usually relies on the idea of "saturation" i.e. collecting data until the point is reached where no new information, ideas or themes are observed in the data ${ }^{[30]}$. Saturation usually occurs within the first twelve interviews and main themes can be present in as early as six interviews ${ }^{[30]}$. In this research, thematic saturation was reached after nine interviews. The transcriptions were analyzed independently by the first two authors utilizing the methods outlined by Aurebach ${ }^{[31]}$. A constant comparative method was utilized, whereby the two researchers compared the themes and where differences occurred, they discussed and came to final agreement. The methods include a number of steps in theory coding, including raw data, relevant text, repeating ideas, themes, theoretical constructs, and theoretical narrative. Once the relevant text had been chosen, repeating ideas were grouped together into one document, which generated a list of $>47$ ideas, including a section on "orphan ideas" (i.e., those that did not necessarily fit into any one idea category but were still deemed important). Some new ideas were added, and some were merged together. Following this step, the repeating ideas were reanalyzed and merged ones that flowed together into themes, thus paving the way for the theoretical narrative that is presented in the Results section.

\subsection{Ethics}

Ethics approval was obtained from the University of Toronto's Research Services Ethics Review Unit. Some hospitals required additional ethical review within the hospital, and this was obtained where necessary.

\section{Results}

Of the nine subjects that were interviewed, eight were registered nurse leaders and one was a non-health-care professional. The length of time that participants held their current positions ranged from six months to 13 years. The types of positions 
held by the participants ranged from performance management reporting coordinator to director of nursing practice. Some subjects held mandated responsibility for CPGs and for others it was an added function, yet all participants had some responsibility for CPGs. Work settings included six community hospitals, one small hospital, and two urban teaching hospitals. Some organizations had affiliated health care organizations such as long-term care facilities or community care access centres (CCACs) that coordinate home care services in Ontario. Overall, a range of hospital settings was represented. Data were not compared by hospital type, but barriers to success seemed common among subjects regardless of setting.

Most hospitals had used some guidelines for some time. The number of CPGs ranged from 2 to 40 and disease states were broad. Some subjects noted that CPGs were both formalized and informal - the latter being "understood" care approaches not formally called CPGs, and not truly tracked by the hospitals. As a result, respondents claimed that more CPGs were used in practice than are actually recorded. Most subjects felt CPGs led to a definite decrease in LOS i.e. "Pneumonia length of stay has decreased by two days ... Our acute coronary syndrome, their length of stay has gone down from about 4 days to 2”. Some interviewees did mention instances where no effect on LOS was observed.

The analysis of the interviews identified five major factors that can influence the impact of CPGs on LOS. These factors help to explain the conflicting findings between the systematic reviews, which found an inverse relationship between CPG usage and LOS and the Ontario hospital data which found minimal relationships. The five main themes were alternateive purposes for the CPG implementation (i.e. reduced LOS was not the goal), the evidence base of the CPG, resistance from healthcare professions to CPG use, poor or inadequate dissemination of CPGs, and lack of organizational support.

\subsection{Alternative purposes for CPG implementation}

Hospitals and unit managers implement CPGs for a variety of reasons, not just to reduce LOS. Hospitals may implement CPGs because of external factors such as pressure from other organizations implementing CPGs (i.e., "It's part of the whole strategic process looking at what the future is ... everybody is moving towards evidence-based practice"), regional healthcare bodies called Local Health Integration Networks (LHINs) (i.e., "Getting the pressure too from the LHINs"), or professional associations (i.e., "We actually have someone who sits on ... a specialty group and general surgery for the province, and so they had actually implemented those”).

Table 1. Effect of CPGs on outcomes (excluding LOS)

\begin{tabular}{|c|c|}
\hline Outcome of interest & Representative citations \\
\hline Improve consistency of care & "That's one of our main objectives ... to ensure consistency ... the consistency is certainly there" \\
\hline Re-admissions & “Our re-admission rate has decreased in heart failure” \\
\hline Quality of care & $\begin{array}{l}\text { "The quality of care has improved ... so many things were being missed before and now they're just } \\
\text { automatically ordered" }\end{array}$ \\
\hline Patient satisfaction & “...things also like patient satisfaction, improvement” \\
\hline Staff satisfaction & $\begin{array}{l}\text { "We're hoping for increased staff satisfaction ... how does this affect the workload or the care } \\
\text { delivery for nurses and allied health?" }\end{array}$ \\
\hline Flow to community & "The flow from hospital to community has improved" \\
\hline
\end{tabular}

Although outside pressures may drive an organization to implement CPGs, the specific CPG disease state selected are usually chosen within the organization. Some hospitals focused their CPGs on high-frequency patient populations and most hospitals decided which clinical area to focus on based on improving their current outcome measures for quality, efficiency, decreasing costs or variations of care. There was no consistent reason why CPGs were being implemented in certain disease states, but it was clear that outcomes other than LOS were also targeted (see Table 1). Thus one possible reason for the lack of a relationship between CPG usage and LOS in the Ontario hospital data is that not all hospitals target LOS as the main outcome variable. 


\subsection{Evidence base for CPG content and selection}

Interviewees reported that for the majority of CPGs they selected existing CPGs developed by well-established national or international organizations or from the literature i.e., "As much as possible, if there are national guidelines, we try to use those”. Participants stated that existing CPGs developed by reliable organizations are preferred to those developed in house because a) there are limited in-house resources to focus on CPG development, and b) outside organizations have a wider reach of experts who can be tapped for their knowledge and expertise.

Although existing CPGs may have been adopted, many of the interviewees commented that the CPG still needed "to be tailored to the institution”. However they recognized that although tailoring a CPG to the institution makes it locally relevant, it also risks making it less effective. Furthermore, once a CPG is adopted, it needs to be evaluated and updated regularly but some participants stated that while the organization requires annual or bi-annual review, it is not always done and is usually an ad hoc process. Updating the CPG and reviewing it - based on new literature, staff feedback, or professional recommendations - ensures that the CPGs represent best practice. By not updating a CPG, the hospital may limit its effect on LOS.

All participants stated that appropriate health care professionals were involved when discussing the development and implementation of a CPG, for example one interviewee said, "we made sure that everybody was at the table... I brought in the people who needed to be at the table, so it was vetted through all of the areas". Some organizations included community care representatives, management, information technology staff, pharmacists, and possibly representatives from each site within the hospital in the development process.

Thus the use of existing CPGs and a multidisciplinary team to develop and approve the CPG is common practice. Considering that CPGs are implemented in a multidisciplinary context, it supports the current thinking of team care and collaborative care and can further enhance the buy in and shared ownership. However, using pre-existing CPGs could limit the impact of the CPG on LOS due to lower efficacy resulting from the adaptation of the CPG for a different environment. Also, the lack of follow-up and revisions of the CPGs could influence the CPG-LOS relationship.

\subsection{Health care professionals' response to change and ensuing compliance}

A third factor influencing the impact of CPGs on LOS is the lukewarm or negative reaction of some health care professionals to the CPGs. If the CPG is "implemented" but not used, its effects are minimal. Some health care professionals comply because a CPG takes some responsibility off them and supports their work i.e., "It was kind of a win-win for the OTs; It off-loaded some of the work that they had to do because they weren't the owners anymore of that" and "most nurses see these as ways of making their practice easier".

Other professionals resisted the implementation of CPGs and the change to their practice. Interviewees, who were largely responsible for implementation, found the lack of change "very frustrating". Table 2 presents some of the main reasons health care professionals do not change their practice to include the CPGs. These include preferring the status quo, not understanding the added value, fear of change and overall workload.

This resistance from staff could be a major barrier to CPGs' efficiency. Physicians were singled out as the group most resistant to change and the most difficult to convince to use the CPGs. For example one interviewee stated: "I would say our biggest barrier has been physicians not wanting to start it, and physicians telling the nurses, 'Don't start this pathway; I don't want this patient on the pathway', because they don't like it”. The reasons specific to physicians' resistance to CPG implementation fell into three main categories: generational issues, autonomy issues, and they did not like practicing “cookie-cutter” medicine (see Table 3). 
Table 2. Reasons for lack of adoption of CPGs by health care professionals

\begin{tabular}{|c|c|}
\hline Reason for poor adoption & Representative citations \\
\hline Prefer status quo & $\begin{array}{l}\text { "It's the way they’ve always learned ... they learned how to do something, and they never moved } \\
\text { beyond that" }\end{array}$ \\
\hline Do not see the value & $\begin{array}{l}\text { "Again, part of it is also attitude. Those people ... who see the value in them are using them. The } \\
\text { ones who don't, avoid them" }\end{array}$ \\
\hline $\begin{array}{l}\text { Constant change/flavour of } \\
\text { the month }\end{array}$ & $\begin{array}{l}\text { "It does get to be a bit of the flavour of the month, and if we could just focus on one thing for a } \\
\text { while ..." }\end{array}$ \\
\hline $\begin{array}{l}\text { Afraid of change and avoid } \\
\text { change }\end{array}$ & “They say, 'If I close my eyes and I don’t look at it, it’s going to go away’” \\
\hline Workload & $\begin{array}{l}\text { "It's all about workload. I think that's the biggest issue that we're having right now in terms of time } \\
\text { and workload to actually sit down and read the CPGs and understand them and implement them } \\
\text { into practice" }\end{array}$ \\
\hline
\end{tabular}

Table 3. Physician resistance to CPGs

\begin{tabular}{|c|c|}
\hline Resistance due to: & Representative citations \\
\hline \multirow{5}{*}{ Generational } & $\begin{array}{l}\text { "This old guard of plastic surgeons ... they are very antiquated in their treatment of wounds; they've been } \\
\text { doing the same thing for } 20 \text { years ... They are very set in their ways...” }\end{array}$ \\
\hline & On the other hand, two interviewees stated that: \\
\hline & "Initially I would have told you that the young ones are more on board, but now that we've gotten into it, I \\
\hline & can't say that anymore. Because some of our older ones, they are on board and they completely believe in \\
\hline & evidence-based medicine. So, initially I probably had an easier time with the young guys but not anymore.” \\
\hline Autonomy & $\begin{array}{l}\text { “A lot of issues with physicians, you know, or 'Not with my patient'. Well, it’s difficult if you can } \\
\text { implement practice guidelines and, you know, if they're saying, 'Well, it counts for everybody except my } \\
\text { patients ...”” }\end{array}$ \\
\hline $\begin{array}{l}\text { Resist "cookie-cutter" } \\
\text { medicine }\end{array}$ & “The internists have been, like, 'Well no one’s going to tell me how to practice cookbook medicine.”” \\
\hline
\end{tabular}

While some health care professionals embrace CPGs from the start, others are slow to buy-in but eventually do, and a large part of the staff are resistant to using CPGs. If the CPGs are implemented for the purpose of reducing LOS but are not used, the effects will not be actualized.

\subsection{Dissemination strategies}

Participants identified another factor limiting the impact of CPGs on length of stay related to the dissemination and implementation process. Proper implementation of a health system innovation can greatly influence its effect on outcomes. Interviewees described implementing the CPG as the most difficult component of the CPG adoption process. Education was the most prevalent method for disseminating CPGs. Only two hospitals did not provide formal education and relied instead on discussions on the units regarding CPG use. All hospitals mentioned some type of educational initiatives, yet some were more thorough than others - a potential reason why there is a limited effect of CPGs on LOS.

A second component of dissemination involves reminding staff of the relevant CPGs and monitoring their use. Reminders were sent through a variety of means, such as e-mails, in-house publications, and posters. In some hospitals, the person most responsible for the CPG implementation regularly reviewed patients' charts to ensure CPG usage. In some hospitals, this included follow up emails, letters or discussions if patients’ care was not guided by a CPG.

Information technology also can promote dissemination. Some hospitals had a highly automated system, in which the CPG was integrated right into the online patient care plans, other hospitals had the CPGs available electronically on the Intranet to peruse or download and included paper forms in the patients' charts, and a few hospitals only had the CPGs available on paper. Many interviewees stated the desire to switch and "go with an automated system" and felt that professionals are not using CPGs as much as possible because they were not electronic. The interviewees perceived that if the CPGs were 
implemented on an electronic system, their adoption would increase i.e., "If it's electronic, then we know it's going to be used because it’s going to be integrated right into their intervention screen”.

A final element regarding effective dissemination mentioned by the interviewees was the need to have the CPG readily available (which reminds staff to use it). One interviewee stated "The CPG is readily available on the unit; there are about six different places you can pick it up on each ward”. The hospitals used a variety of techniques to implement the CPGs and all hospitals included education as a central component. However, each interviewee mentioned only some of the initiatives. So the limited impact of CPGs on LOS could be increased if the hospitals undertook additional integrated dissemination activities to ensure compliance.

\subsection{Organizational support and resources}

Organizational support also influences the impact of CPGs on LOS. Some interviewees felt that their organization did not fully support the interventions since there was no accountability for change. One interviewee stated "We don't have very much continuity of care ... because no one ever really has to be accountable for what they're doing because it's only what they did that day".

The interviewees felt frustrated that their organizations did not fully support the change: "the whole organization needs to turn around and think about what needs to be done to support that front-line worker" in using CPGs. Many interviewees wished for "leadership buy-in and support to promote and sustain CPGs, because what I find is that we got it initially, but the attention isn't there. They're off doing something else, or they're not focused on clinical pathways anymore”.

The culture of the organization could also facilitate or impede CPG uptake, and some participants used their culture to their advantage i.e., "I think that as an academic health science centre, we've certainly got a culture which has respect for evidence-based practice and we're really pushing that”. Other interviewees recognized that the culture of the organization needed to shift to support the change, "How do you influence the culture so that you're moving from the traditional paradigm of the medical model and management techniques to autonomy in practice and having staff nurses and others take on the responsibility independently?”

Some interviewees also felt that their organization had not allocated sufficient funds for dedicated education and support, "I think that should be incorporated in the budget planning... Having those opportunities to do it right, that you're not scrimping and saving, you're not trying to beg, borrow, or steal dollars to do it as a piecemeal thing”. Several participants claimed that there are too few resources in place to support the CPG-uptake process - this may be one of the largest impediments. There was an overall sense of the lack of resources: "You do need a lot more resources available to support the implementation”. Indeed only one participant stated that developing, implementing, and monitoring CPGs was her full-time job. For most interviewees, this role was an additional responsibility to their other jobs, "There's not a dedicated person ... it would have been fabulous to have one sort of person, not an add-on to her role”.

These quotations illustrate the limited human resources available to implement and lead the CPG process. From photocopying the CPGs and disseminating them on the floors, to the high-level strategic planning and implementation, the interviewees felt that they were responsible for substantial changes above their existing roles. Interviewees repeatedly emphasized the need for more resources, both human and financial, if their organizations wanted to realize positive outcomes from CPGs.

\section{Discussion}

Hospitals in this study implemented CPGs for many reasons and undertook significant planning in selecting CPGs and educating staff. The effectiveness of CPGs may be impacted by the nature of the CPG used, but five categories of organizational and individual level factors emerged during the interviews that could explain the relationships between 
CPGs and LOS. These five factors include goals for CPG use that do not include reductions in length of stay, adaptation of the CPG in ways that undermine its effectiveness, resistance from healthcare professions to CPG use, poor or inadequate dissemination of CPGs, and lack of organizational support.

\subsection{Relation to other finding}

A study examining over 700 CPGs in the Canadian Medical Association database found that CPGs are usually evidencebased ${ }^{[32]}$, and have become more so over time ${ }^{[33]}$. Most hospitals do not have the resources to thoroughly develop CPGs leaving pre-existing CPGs as the most feasible option ${ }^{[34]}$. Even when adopting existing CPGs, many interview subjects confirmed earlier findings that CPGs need to be adapted to local factors within each institution to promote uptake ${ }^{\text {[34-37] }}$. However, this research demonstrates that adaptation could impede the CPG-LOS relationship.

Previous research has identified the lack of clinician willingness to change as a barrier. For example, Solberg and colleagues identified organizational and change management characteristics as the main barriers to CPG implementtation ${ }^{[38-41]}$. Some providers comply quickly because CPGs relieve them of some responsibility and support their work, while others are slower to change practice or resist outright. Yet, consistent with findings in change management literature ${ }^{[42]}$, some interview subjects found that providers became more engaged over time. Evidence of the impact of CPGs might assist in their adoption ${ }^{[43]}$, yet data about CPG benefits and outcomes were rarely distributed to the staff in this study, even when evaluation results were available. "Proving value of the 'new' way to others" is an important part of the change process and is rarely done, according to Reay and colleagues ${ }^{[42]}$. This last component of the CPG process needs to occur to ensure success.

The involvement of multi-disciplinary team members can affect compliance ${ }^{[34,35,44]}$ and participants in this study did feel that multi-disciplinary teams assisted in the success of the CPG. However, certain disciplines were quite resistant to the implementation of the CPGs. Interview subjects noted particular resistance among physicians, related mainly to age/generation, autonomy issues, and resistance to practicing "cookie-cutter" medicine. Other studies found varying reactions among clinicians to CPGs. One study that focused specifically on Ontario cancer physicians found positive attitudes toward CPGs, but other researchers found resistance ${ }^{[45-47]}$. As revealed in the current interviews, physicians are hardly alone in their resistance to change. Furthermore, without monitoring of compliance, managers cannot target specific interventions or groups to promote usage.

The complexity and difficulty of implementing CPGs has been identified in several studies ${ }^{[19,21,23,48,49]}$, a finding mirrored in this research. Education and subsequent reminders encourage uptake and facilitate change ${ }^{[10,20,21,50,51]}$. Other methods for implementing and disseminating CPGs to facilitate compliance are identified in the literature ${ }^{[21,24,32]}$. Most prevalent were various approaches to educating staff about CPGs. However, in this study interview subjects mentioned only some of these activities. Thus the lack of relationship between CPGs and LOS could be undermined because hospitals fail to undertake broader dissemination strategies to ensure compliance.

Organizational support is another critical element the influencing the impact of CPGs on LOS. Such support was inconsistent across the hospitals studied. For CPGs to be implemented successfully, the organizational climate and context must be open to change ${ }^{[50,52,53]}$; Moreover an "organizational approach to change management and quality improvement", and an assurance that "adequate resources have been committed" to the work are essential ${ }^{[38]}$. Failure to attend to these issues may create an important barrier to the relationship between CPGs and LOS. The findings in this study are consistent with previous studies and assist in further understanding the specific issues and barriers in the Canadian context.

\subsection{Strengths and limitations}

There are two main strengths to this study which were a) there was limited variation in participants' responses by organization and b) saturation was reached, i.e. the participants were encountering similar barriers and facilitators to CPG dissemination and implementation. There were two main limitations to this study which were: a) the study was confined to 
a certain position type, i.e., employees who oversee CPG dissemination and implementation, and other position types might have had other perspectives, and b) the study was only conducted in one province and generalizability may be limited.

\subsection{Future research}

While this research contributes to the understanding of the opinions of those that lead the implementation of CPGs, future research should include those involved in different stages of the CPG process and who actually treat patients using CPGs. Such interviews could examine why staff use or do not use CPGs, how they perceive the effects, and what barriers they encounter. To gauge the enduring impacts of CPGs, a broader set of interviews with subjects beyond nursing, in more settings, and at different points in the CPG process might prove valuable. Further research could also examine the job characteristics of individuals assigned primarily with CPG responsibilities and the time allotted to the task. This strategy may illuminate the types of human resources needed to implement CPGs.

\section{Conclusion}

Implementing CPGs is an important but challenging undertaking. The difficulty of the task is magnified when appropriate organizational supports are not in place and barriers to implementation occur. Dedicated individual efforts seem to be responsible for many of the accomplishments achieved to date. But for changes to be institutionalized and become system-wide standards of care, more research and support are needed. Furthermore it is essential to continue assessing the value of these approaches to understand their impact on patients, providers and systems.

In summary, numerous factors could cause CPGs to negatively or positively impact LOS. Grol and Grimshaw (2003) identify barriers to change at many different levels within an organization and with patients or employees. In this research, professional barriers were exhibited in the resistance of providers to change, and organizational barriers are seen in the lack of human and financial resources.

If the organizations represented in this study are typical, far fewer resources are being spent on implementation than development of CPGs. If their benefits are to be realized, more effort must be focused on effective strategies to promote uptake ${ }^{[32]}$. While many prescriptive articles discuss ways to implement CPGs effectively ${ }^{[21,23,54]}$, the responses in this study suggest that these efforts are not well integrated with other work.

Since different hospitals encounter distinct types of barriers depending on their organizational cultures, their environments, and their individual practitioners every hospital requires different implementation strategies and resources $^{[34,55]}$. One common barrier in CPG implementation is overcoming the "inertia of previous practice" ${ }^{\text {[49]; }}$ There is a strong attachment to past ways of delivering patient care ${ }^{[43]}$. Unfreezing of old patterns needs to occur so that change can be introduced, and the new way of practice has to be "refreezed" ${ }^{[55]}$.

\section{Acknowledgments}

Dr. Ellen was supported by a Canadian Institutes for Health Research Doctoral Research Award.

\section{References}

[1] Beech R, Larkinson J. Estimating the financial savings from maintaining the level of acute services with fewer hospital beds. International Journal of Health Planning \& Management. 1990; 5: 89-103. http://dx.doi.org/10.1002/hpm.4740050203

[2] Robertson ML, Edmond BJ, Lindsey JO, Vallejo BC. Reducing length of stay through rapid clinical redesign. Journal for Healthcare Quality. 2002; 24: 26-31. PMid: 12240540. http://dx.doi.org/10.1111/j.1945-1474.2002.tb00457.x 
[3] Dy SM, Garg PP, Nyberg D, Dawson PB, Pronovost PJ, Morlock L, et al. Are critical pathways effective for reducing postoperative length of stay? Medical Care. 2003; 41: 637-648. PMid: 12719688. http://dx.doi.org/10.1097/01.MLR.0000062552.92534.BE

[4] Rotter T, Kinsman L, James E, Machotta A, Gothe H, Willis J, et al. Clinical pathways: effects on professional practice, patient outcomes, length of stay and hospital costs. Cochrane Database Syst Rev. 2010. PMid: 20238347. http://dx.doi.org/10.1002/14651858.CD006632.pub2

[5] Field MJ, Lohr KN. Clinical Practice Guidelines: Direction of a New Program. Washington, DC: National Academy Press. 1990.

[6] Woolf SH, Grol RP, Hutchinson A, Eccles M, Grimshaw JM. Potential benefits, limitations, and harms of clinical guidelines. British Medical Journal. 1999; 318: 527-530. PMid: 10024268. http://dx.doi.org/10.1136/bmj.318.7182.527

[7] Grimshaw JM, Russell IT. Effect of clinical guidelines on medical practice: A systematic review of rigorous evaluations. Lancet. 1993; 342: 1317-1322. http://dx.doi.org/10.1016/0140-6736(93)92244-N

[8] Ellen ME. Examining The Relationship Between The Use Of Clinical Practice Guidelines And Hospital Length Of Stay: A Systematic Review. 2007.

[9] Ellen ME, Brown A, Cockerill R. Do clinical practice guidelines influence length of stay in Ontario acute care hospitals? Healthcare Quarterly. 2009; 12: 64-71. PMid: 20057231. http://dx.doi.org/10.12927/hcq.2013.21128

[10] Davis DA, Taylor-Vaisey A. Translating Guidelines into Practice. Canadian Medical Association Journal. 1997; 157: 408-416. PMid: 9275952.

[11] Alonso-Coello P, Irfan A, Sola I, Gich I, Delgado-Noguera M, Rigau D., et al. The quality of clinical practice guidelines over the last two decades: a systematic review of guideline appraisal studies. BMJ. 2010; 19: 1-7.

[12] AGREE Enterprise. AGREE: Advancing the science of practice guidelines. Available from: http://www.agreetrust.org/resource-centre/practice-guidelines/. 2013; 24-10-2013. Ref Type: Electronic Citation

[13] Guidelines Advisory Committee. Guidelines Advisory Committee Website. www.gacguidelines.ca. 2005. Ref Type: Electronic Citation

[14] Shaneyfelt TM, Mayo-Smith MF, Rothwangl J. Are guidelines following guidelines? The methodological quality of clinical practice guidelings in the peer-reviewed medical literature. Journal of American Medical Association. 1999; 281: $1900-1905$. http://dx.doi.org/10.1001/jama.281.20.1900

[15] Grilli R, Magrini N, Penna A, Mura G, Liberati A. Practice guidelines developed by specialty societies: The need for a critical appraisal. Lancet. 2000; 355: 103-106. http://dx.doi.org/10.1016/S0140-6736(99)02171-6

[16] Fervers M, Burgers JS, Haugh MC, Brouwers M, Browman G, Cluzeau F, et al. Predictors of high quality clinical practice guidelines: examples in oncology. International Journal for Quality in Health Care. 2004; 17: 123-132. PMid: 15665068. http://dx.doi.org/10.1093/intqhc/mzi011

[17] Brouwers M, Kho ME, Browman GP, Burgers J, Cluzeau F, Feder G, et al. Performance, Usefulness and Areas for Improvement: Development Steps Towards the AGREE II - Part 1. Canadian Medical Association Journal. 2010; 182: 1045-1052. PMid: 20513780. http://dx.doi.org/10.1503/cmaj.091714

[18] Brouwers M, Kho ME, Browman GP, Burgers JS, Cluzeau F, Feder G, et al. AGREE II: Advancing guideline development, reporting and evaluation in healthcare. Canadian Medical Association Journal. 2010. http://dx.doi.org/10.1503/cmaj.090449

[19] Grol R, Grimshaw J. From Best Evidence to Best Practice: Effective Implementation of Change in Patients' Care. The Lancet. 2003; 362: 1225-1230. http://dx.doi.org/10.1016/S0140-6736(03)14546-1

[20] Grol RP, Grimshaw JM. From best evidence to best practice: Effective implementation of change in patients' care. The Lancet. 2003; 362: 1225-1230. http://dx.doi.org/10.1016/S0140-6736(03)14546-1

[21] Grimshaw JM, Shirran L, Thomas R, Mowatt G, Fraser C, Bero L, et al. Changing Provider Behavior: An Overview of Systematic Reviews of Interventions. Medical Care. 2001; 39 (suppl 2): 2-45. http://dx.doi.org/10.1097/00005650-200108002-00002

[22] Grimshaw JM, Shirran L, Thomas R, Mowatt G, et al. Getting Evidence into Parctice. NHS Centre for Reviews and Dissemination, Cochrane Effective Practice and Organisation of Care Group: Effective Health Care. 1999; 5.

[23] Grimshaw JM, Eccles MP, Walker AE, Thomas RE. Changing Physician' Behavior: What Works and Thoughts on Getting More Things to Work. The Journal of Continuing Education in the Health Professions. 2002; 22: 237-243. PMid: 12613059. http://dx.doi.org/10.1002/chp.1340220408

[24] Grimshaw J, Thomas RE. Effectiveness and Efficiency of Guideline Dissemination and Implementation Strategies. Health Technology Assessment. 2004; 8.

[25] Turner T, Misso M, Harris C, Green S. Development of evidence-based clinical practice guidelines (CPG's): comparing approaches. Implement Sci. 2008; 3.

[26] Gundersen L. The Effect of Clinical Practice Guidelines on Variations in Care. Annals of Internal Medicine. 2006; 133: 317-318. http://dx.doi.org/10.7326/0003-4819-133-4-200008150-00102 
[27] Morse J, Field P. Qualitative Research Methods for Health Professionals. Thousand Oaks, Sage. 1995.

[28] Marshall C, Rossman G. Designing Qualitative Research. Newbury Park: Sage. 1989.

[29] Onwueqbuzie AJ, Collins K. A Typology of Mixed Methods Sampling Designs in Social Science Research. Qualitative Report. 2007; 12: 281-316.

[30] Guest G, Bunce A, Johnson L. How Many Interviews Are Enough? An Experiment with Data Saturation and Variability. Field Methods. 2006; 18: 59-82. http://dx.doi.org/10.1177/1525822X05279903

[31] Aurebach CF. Qualitative Data: An Introduction to Coding and Analysis. New York: New York University Press. 2003.

[32] Graham ID, Beardall S, Carter AO, Tetroe J, Davies B. The State of the Science and Art of Practice Guidelines Development, Dissemination, and Evaluation in Canada. Journal of Evaluation in Clinical Practice. 2003; 9: 195-202. PMid: 12787183. http://dx.doi.org/10.1046/j.1365-2753.2003.00385.x

[33] Clancy CM, Cronin K. Evidence-Based Decision Making: Global Evidence, Local Decisions. Health Affairs. 2005 ; $24:$ 151-162. PMid: 15647226. http://dx.doi.org/10.1377/hlthaff.24.1.151

[34] Feder G, Eccles M, Grol R, Griffiths C, Grimshaw J. Using Clinical Guidelines. BMJ. 1999; 318: 728-730. PMid: 10074024. http://dx.doi.org/10.1136/bmj.318.7185.728

[35] Graham ID, Harrison MB, Brouwers M, Davies BL, Dunn S. Facilitating the Use of Evidence in Practice: Evaluating and Adapting Clinical Practice Guidelines for Local Use by Health Care Organizations. Journal of Obstetric, Gynecological, and Neonatal Nursing. 2002; 31: 599-611. http://dx.doi.org/10.1111/j.1552-6909.2002.tb00086.x

[36] Harrison MB, Legare F, Graham I, Fervers B. Adapting Clinical Practice Guidelines to local context and assessing barriers to their use. Canadian Medical Association Journal. 2010; 182: E78-84. PMid: 19969563. http://dx.doi.org/10.1503/cmaj.081232

[37] Silagy CA, Weller DP, Lapley H, Middleton P, Shelby-James P, Fazekas B. The effectiveness of local adaptation of nationally produced clinical practice guidelines. Family Practice. 2002; 19: 223-230. PMid: 11978710. http://dx.doi.org/10.1093/fampra/19.3.223

[38] Solberg LI, Brekke ML, Fazio CJ, Fowles J, Jacobsen DN, Kottke TE, et al. Lessons from Experienced Implementers: Attend to Many Factors and Use Multiple Strategies. Journal on Quality Improvement. 2000; 26: 171-188.

[39] Dijkstra R, Wensing M, Thomas R, Akkermans R, Braspenning J, Grimshaw J, et al. The relationship between organisational characteristics and the effects of clinical guidelines on medical performance in hospitals: A meta-analysis. BMC Health Services Research. 2006; 6.

[40] Scott T, Mannion R, Marshall M, Davies HTO. Does organisational culture influence health care performance? A review of the evidence. Journal of Health Services Research and Policy. 2003; 8: 105-117. PMid: 12820673. http://dx.doi.org/10.1258/135581903321466085

[41] Ward MM, Yankey JW, Vaughn TE, Bootsmiller BJ, Flach SD, Watrin S, et al. Provider adherence to COPD guidelines: relationship to organizational factors. Journal of Evaluation in Clinical Practice. 2005; 11: 379-387. PMid: 16011650. http://dx.doi.org/10.1111/j.1365-2753.2005.00541.x

[42] Reay T, Golden-Biddle K, Germann K. Legitimizing a New Role: Small Wins and Microprocesses of Change. Academy of Managament Journal. 2006; 48: 977-998. http://dx.doi.org/10.5465/AMJ.2006.22798178

[43] Cabana MD, Rand CS, Powe NR, Wu AW, Wilson MH, Abboud PC, et al. Why Don't Physicians Follow Clinical Practice Guidelines? A Framework for Improvement. Journal of the American Medical Association. 1999; 282: 1458-1465. PMid: 10535437. http://dx.doi.org/10.1001/jama.282.15.1458

[44] Pagliari C, Grimshaw J, Eccles M. The Potential Influence of Small Group Processes on Guideline Development. Journal of Evaluation in Clinical Practice. 2001; 7: 165-173. PMid: 11489041. http://dx.doi.org/10.1046/j.1365-2753.2001.00272.x

[45] Graham ID, Brouwers M, Davies C, Tetroe J. Ontario Doctors' Attitudes Toward and use of Clinical Practice Guidelines in Oncology. Journal of Evaluation in Clinical Practice. 2006. PMid: 16579826.

[46] Farquhar C, Kofa E, Slutsky J. Clinicians' Attitudes to Clinical Practice Guidelines: A Systematic Review. Medical Journal of Australia. 2002; 177: 502-506. PMid: 12405894.

[47] Cabana MD, Rand CS, Powe NR, Wu AW, Abboud PA, Rubin HR. Why don't physicians follow clinical practice guidelines? A framework for improvement. Journal of the American Medical Association. 1999; 282: 1458-1465. PMid: 10535437. http://dx.doi.org/10.1001/jama.282.15.1458

[48] Santamaria N, Houghton L, Kimmel L, Graham A. Clinical pathways for fractured neck of femur: a cohort study of health related quality of life, patient satisfaction and clinical outcome. Australian Journal of Advanced Nursing. 2003 Mar-May; 20(3): 24-9.

[49] Shiffman RN, Michel G, Essaihi A, Thornquist E. Bridging the Guideline Implementation Gap: A Systematic, Document Centered Approach to Guideline Implementation. Journal of the American Medical Informatics Association. 2004; 11: 418-426. PMid: 15187061. http://dx.doi.org/10.1197/jamia.M1444 
[50] Meijers JMM, Janssen MAP, Cummings GG, Wallin L, Estabrooks CA, Halfens RYG. Assessing the Relationships Between Contextual Factors and Research Utilization in Nursing: Systematic Literature Review. Journal of Advanced Nursing. 2006; 55: 622-635. PMid: 16907795. http://dx.doi.org/10.1111/j.1365-2648.2006.03954.x

[51] Friedman L, Engelking C, Wickham R, Harvey C, Read M, Whitlock KB. The EDUCATE Study: A Continuing Education Exemplar for Clinical Practice Guideline Implementation. Clinical Journal of Oncology Nursing. 2009; 13.

[52] Devlin R, Czaus M, Santos J. Registered Nurses Association of Ontario's Best Practice Guideline as a Tool for Creating Partnerships. Hospital Quarterly. 2002; Spring: 62-65. PMid: 12055869.

[53] Lanier DC, Roland M, Burstein H, Knottnerus JA. Doctor Performance and Public Accountability. The Lancet. 2003; 362: 1404-1408. http://dx.doi.org/10.1016/S0140-6736(03)14638-7

[54] DiCenso A, Virani T, Bajnok I, Broycki E, Davies B, Graham I, et al. A Toolkit to Facilitate the Implementation of Clinical Practice Guidelines in Healthcare Settings. Hospital Quarterly. 2002; Spring: 55. PMid: 12055868.

[55] The University of York: Effective Health Care: Getting Evidence into Practice. NHS Center for Reviews and Dissemination. 1999; 5. 